\title{
Invasive and non-invasive group A $\beta$-haemolytic streptococcal infections in patients attending public sector facilities in South Africa: 2003-2015
}

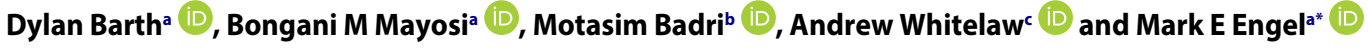

\author{
a Department of Medicine, University of Cape Town and Groote Schuur Hospital, Cape Town, South Africa \\ ${ }^{b}$ College of Medicine, King Saudi Bin Abdulaziz University for Medical Sciences, Riyadh, Kingdom of Saudi Arabia \\ 'Department of Microbiology, National Health Laboratory Service, Tygerberg Hospital and Stellenbosch University, Cape Town, South Africa \\ *Corresponding author, email: mark.engel@uct.ac.za

\begin{abstract}
Background: The burden of disease caused by group A streptococcus (GAS) in Africa is largely unknown. The aim of this study was to determine the incidence of invasive (iGAS) and non-invasive GAS (non-iGAS) infections in patients attending the public health sector of South Africa.

Methods: iGAS and non-iGAS infection was defined as GAS isolated in culture from sterile and non-sterile sites respectively. Using annual census data, we calculated incidence rates (IR) of $i$ GAS and non-iGAS infection by reviewing cases from the National Health Laboratory Service (NHLS) database derived from the 9 provinces of South Africa between 2003 and 2015.

Results: About $50 \%$ of the samples were collected in the Eastern Cape province which had data for all the years under observation; missing data from the other eight provinces precluded analysis of annual incidence. A multi-modal distribution of 5256 GAS cases was observed in the Eastern Cape province. iGAS cases $(n=428)$ showed an annual mean IR of 0.48 (Range: $0.15-1.12$ ) cases per $10^{5}$ per year (py) with a marginal decrease from 2003 to 2015 (Rate Difference (RD), 0.23/10 $\mathrm{py}$; $95 \%$ Cl: $0.02-0.44 / 10^{5}$ py). The mean annual IR for non-iGAS infection $(n=4828)$ was 5.48 (Range: $0.19-11.55)$ cases/10 $10^{5}$; IR showed a decrease $\left(R D, 11.36 / 10^{5}\right.$ py; $95 \% \mathrm{Cl}: 10.53-12.19 / 10^{5}$ py). The Mann-Kendall test and the Theil-Sen estimator showed a decreasing trend in the incidence of non-iGAS infection $(p=0.002)$ over the study period.

Conclusions: The incidence of non-iGAS infection in the Eastern Cape province of South Africa declined from 2003 to 2015 . The trends from the Eastern Cape and incomplete data from other provinces indicate the need for a detailed prospective evaluation of GAS infection in South Africa to verify this trend and provide information for planning appropriate interventions.
\end{abstract}

Keywords: epidemiology, group A streptococcus, incidence, infectious disease, public sector, South Africa

\begin{abstract}
Introduction
Streptococcus pyogenes, also known as group A $\beta$-haemolytic streptococcus (GAS), is responsible for a wide range of invasive and non-invasive diseases. ${ }^{1,2}$ These diseases range from mild infections such as impetigo and pharyngitis to serious diseases such as streptococcal toxic shock syndrome (STSS) and necrotising fasciitis. Moreover, GAS may trigger autoimmune diseases following repeated episodes of infection, such as acute rheumatic fever (ARF) and subsequent rheumatic heart disease (RHD) and acute post-streptococcal glomerulonephritis (APSGN). ${ }^{3}$
\end{abstract}

Increases in the annual number of cases for invasive GAS (iGAS) and non-invasive GAS (non-iGAS) diseases have been observed globally since the 1980s. In the United Kingdom, a report from routine surveillance data indicate a significant increase of invasive GAS isolates from December $2008(n=143)$ compared to the same period in $2007(n=86) .{ }^{4}$ The reasons for these observations are not clearly understood and have subsequently caused many countries to commence active surveillance systems for invasive GAS to document the epidemiology of the disease. However, data from the Centres for Disease Control and Prevention surveillance programme reported stable incidence rates for invasive GAS disease from 1996-1999, and from 2002-2009.,

The incidence and prevalence of both invasive and non-invasive GAS infections in developing countries are largely unknown. ${ }^{7}$ Systematically collected data are essential for a functioning disease-control programme ${ }^{8}$ and, thus, the measurement of incidence and temporal trends are an essential first step toward reducing the burden of GAS disease in developing countries. ${ }^{9}$ To the best of our knowledge, there is no surveillance programme for GAS infections in Africa. The aim of this study was to describe the epidemiology of $i$ GAS and non-iGAS infection and associated clinical characteristics in the public sector of South Africa, over a recent 13-year period (2003-2015).

\section{Method}

\section{Study design and population}

This study was conducted under the auspices of the AFROStrep Study, an initiative aimed at documenting the epidemiology of GAS in Africa. ${ }^{10}$ We performed a retrospective analysis of invasive GAS isolates (iGAS) and non-invasive GAS isolates (non-iGAS) cultured at the National Health Laboratory Service (NHLS) from January 2003 to December 2015. The NHLS is the largest diagnostic pathology service in South Africa, supporting both national and provincial health departments in healthcare delivery in all nine provinces of South Africa and is estimated to provide diagnostic pathology services to approximately $80 \%$ of the population (https://www.nhls.ac.za).

\section{Case definition}

iGAS was defined as GAS isolated in culture from a sterile site including blood, cerebrospinal fluid (CSF) and pleural fluid ${ }^{11,12}$. 'Other sites' for invasive infection included GAS isolated from peritoneal fluid, bone, clotted blood, dialysis fluid, serum fluid, hydrocele fluid and deep-tissue specimens. 
GAS isolated from a non-sterile site, such as the skin and throat, was considered to be non-invasive. ${ }^{13}$ 'Other sites' for non-invasive infection included GAS isolated from bronchial swabs, cervical swabs, corneal scraping, nasopharyngeal swabs, faeces, gastric specimens and genital swabs. In addition to microbiological data (including site of isolation), we also abstracted demographic information and clinical data, where available.

\section{Statistical analysis}

The overall and age-specific incidence rates, reported per $10^{5}$ person-years (py), of $i$ GAS and non-iGAS infection were calculated using annual census population data for the Eastern Cape only, given that it represents $52 \%$ of GAS cases in South Africa and its consistent reporting practices in this province. Rate differences were also calculated.

An exploratory statistical analysis was also conducted to identify changes in the trend of GAS infection (increasing, decreasing or remain unchanged) over the 13-year time interval. The change in GAS infection was not expected to be linear from year to year (with no correlation between measurements collected at different times). We, therefore, employed the Mann-Kendall Test for Monotonic Trend (MK test) ${ }^{14}$ rather than parametric linear regression analysis which requires that the residuals from the fitted regression line be normally distributed, an assumption not required by the MK test. Linear trend (median drop per year in GAS infection rates) was assessed using the robust linear regression Theil-Sen estimator; ${ }^{15}$ this technique was shown to be significantly more accurate than simple linear regression for skewed and heteroskedastic data and compares well against non-robust least squares even for normally distributed data in terms of statistical power. The strength of the trend was assessed using the MK test statistic. The test for significance was set at the $5 \%$ level. All statistical analyses were performed using STATA ${ }^{\oplus}$ version 11 (StataCorp, Texas, USA).

\section{Ethical approval}

Ethics approval was obtained from the Human Research Ethics Committee at the University of Cape Town (HREC/REF: R006/2015).

\section{Results}

\section{Clinical characteristics of patients}

The clinical characteristics of patients with iGAS infection and non-iGAS are presented in Table 1. For the 2003 to 2015 period, 10091 GAS isolates were recovered from South African patients. Sources of isolates included blood, CSF, abscesses, joint aspirates,
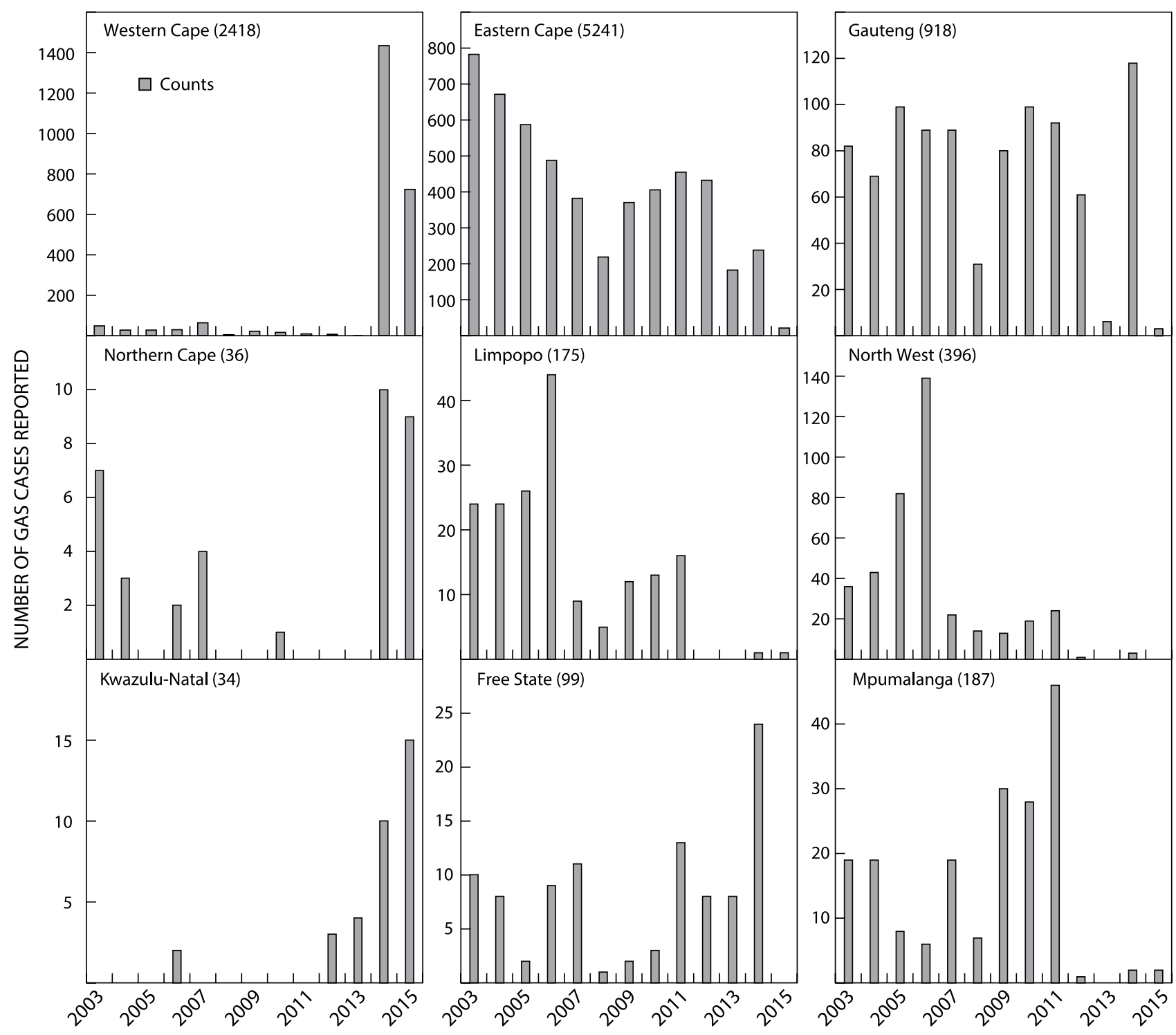

Figure 1: GAS isolation by Province. 
Table 1: Year of isolation, age and sex of the 10091 cases with invasive and non-invasive group A streptococcal infection, South Africa, 20032015

\begin{tabular}{|l|c|}
\hline Year of isolation & $N(\%)$ \\
\hline 2003 & $1079(10.7)$ \\
\hline 2004 & $915(9.1)$ \\
\hline 2005 & $931(9.2)$ \\
\hline 2006 & $959(9.5)$ \\
\hline 2007 & $640(6.3)$ \\
\hline 2008 & $301(3.0)$ \\
\hline 2009 & $571(5.7)$ \\
\hline 2010 & $628(6.2)$ \\
\hline 2011 & $683(6.8)$ \\
\hline 2012 & $521(5.2)$ \\
\hline 2013 & $213(2.1)$ \\
\hline 2014 & $1863(18.5)$ \\
\hline 2015 & $786(7.8)$ \\
\hline Patient age, years & \\
\hline$<18$ & $2920(29)$ \\
\hline $19-64$ & $5902(59)$ \\
\hline$\geq 65$ & $1199(12)$ \\
\hline Sex\# & $5080(51.4)$ \\
\hline Male & $809(48.6)$ \\
\hline Female & \\
\hline & \\
\hline
\end{tabular}

N: number; GAS: Group A Streptococcus.

\#Where data were reported.

Table 2: Source of isolates taken from the 10091 patients with invasive and non-invasive group A streptococcal infection, South Africa, 20032015

\begin{tabular}{|c|c|}
\hline Source of Isolate & $N(\%)$ \\
\hline Invasive GAS* & $1646(16)$ \\
\hline Other aspirates & $194(1.9)$ \\
\hline Joint aspirate & $41(0.4)$ \\
\hline Blood culture & $900(8.9)$ \\
\hline Cerebrospinal fluid & $63(0.6)$ \\
\hline Pleural fluid & $121(1.2)$ \\
\hline Biopsy & $9(0.1)$ \\
\hline Intra-abdominal fluid & $36(0.4)$ \\
\hline Other sites ( $\leq 5$ cases) & $282(2.8)$ \\
\hline Non-invasive GAS* & $8445(84)$ \\
\hline Eye & $56(0.6)$ \\
\hline Vaginal swab & $72(0.7)$ \\
\hline Throat swab & $82(0.8)$ \\
\hline Wound swab & $147(1.5)$ \\
\hline Sputum & $142(1.4)$ \\
\hline Ear swab & $366(3.6)$ \\
\hline Burn swab & $474(4.7)$ \\
\hline Pus swab & $6421(63.6)$ \\
\hline \multicolumn{2}{|l|}{ Urine } \\
\hline \multirow[t]{2}{*}{ Other sites ( $\leq 5$ cases) } & $562(5.6)$ \\
\hline & $123(1.2)$ \\
\hline
\end{tabular}

*Data shown for sites where number of isolates $>5$ per site.

N: number; GAS: Group A Streptococcus. pleural fluid, burn swabs, ear, eye and pus swabs (Table 2). Blood and aspirates were common sources of isolation among iGAS infections, and pus swabs were the most common source of isolation for non-iGAS infections.

\section{Provincial distribution of isolates in South Africa}

When considering the GAS data at a provincial level, a number of abnormal data collection trends were highlighted. The Northern Cape province represented $0.4 \%$ of the total GAS infections in South Africa over the study period; data were missing for years 2005, 2008-2009, 2011-2013 (Figure 1). In the Western Cape, low reporting rates were seen for the first 11 years of the study period, followed by major peaks in reporting rates in 2014 and 2015 (Figure 1). In Gauteng, the overall number of GAS reported over the study period were low considering it has the largest population compared to other provinces in South Africa. The very low number of cases in 2013 and 2015 is also notable (Figure 1). The Free State province showed low reporting of GAS in 2005, 2008-2011 (Figure 1).

The provinces of Limpopo and the North West show similar patterns of reporting with many cases reported in the beginning of the study period followed by a decline after 2006 and almost no data reported after 2011 (Figure 1). The Mpumalanga province also showed inconsistent reporting with a peak in 2011, followed by almost no data thereafter (Figure 1). Kwazulu-Natal recorded the least number cases of GAS in South Africa $(n=34)$ despite it being home to the second largest population, after Gauteng. It must be noted that the Kwazulu-Natal province was the last to be integrated into the NHLS electronic system, with reporting only commencing in the later years (Figure 1). The Eastern Cape province accounted for $52 \%$ of the total GAS cases throughout the country (Figure 1).

\section{GAS disease incidence in South Africa}

The provincial level data revealed a low ascertainment and inconsistent reporting practices of GAS infections throughout the study period. Hence, we were unable to determine any variation in IRs of GAS over the study period across the provinces. The Eastern Cape was the only province with complete data for each of the years within the study period, thus making it possible to evaluate incidence rates and describe trends of GAS infection over the study period.

\section{GAS disease incidence in the Eastern Cape}

The mean annual IR for GAS infection was 6.00 cases per $10^{5}$ py. The annual IR decreased significantly from 2003 to 2015 from $12.04 / 10^{5}$ py to $0.45 / 10^{5}$ py (rate difference (RD): $11.59 / 10^{5} ; 95 \%$ $\mathrm{Cl}: 10.73 / 10^{5}-12.45 / 10^{5}$ ) (Figure 2). Twenty-eight percent of isolates $(n=1474)$ were from patients younger than 18 years of age; $58 \%(n=3014$ ) from patients aged $18-64$ years; and, $14 \%$ $(n=722)$ from those older than 64 years. The mean age was 30 years (Range: 2 days -102 years). The mean age-specific IRs were as follows: $<18$ years: $3.62 / 10^{5}$ py; $18-64$ years: $7.29 / 10^{5}$ py; and, $\geq 65$ years: $14.06 / 10^{5}$ py. The increase in IRs with age was apparent in both the $i G A S$, and non-iGAS sub-sets of patients (Table 3).

\section{Invasive GAS (Eastern Cape)}

Four hundred and twenty-eight (428) cases of $i$ GAS isolates (8\% of total GAS cases in the Eastern Cape) were documented in the NHLS database. A multi-modal curve was observed over the study period (Figure 3 ), with a mean annual IR of 0.48 cases per $10^{5}$ py (mean age: 27 years; Range: 2 days - 84 years). 
Table 3: Mean age-specific incidence rates of GAS, Eastern Cape, 2003-2015

\begin{tabular}{|l|c|c|c|c|}
\hline \multirow{2}{*}{} & \multicolumn{3}{|c|}{ Age Category (yr) } & Total \\
\cline { 2 - 5 } & $<18$ & $18-64$ & $\geq 65$ & Mean IR \\
\hline All GAS $n$ (\%) & $1474(28)$ & $3014(58)$ & $722(14)$ & $5210(100)$ \\
\hline IR (per 10 $0^{5}$ py) & 3.62 & 7.29 & 14.06 & 6.00 \\
\hline $\begin{array}{l}\text { Invasive GAS } \\
n \text { (\%) }\end{array}$ & $153(36)$ & $214(51)$ & $53(13)$ & $420(100)$ \\
\hline IR (per 105 py) & 0.38 & 0.52 & 1.03 & 0.48 \\
\hline $\begin{array}{l}\text { Non-invasive } \\
\text { GAS } n(\%)\end{array}$ & $1321(28)$ & $2800(58)$ & $669(14)$ & $4790(100)$ \\
\hline IR (per 105 py) & 3.24 & 6.77 & 13.03 & 5.48 \\
\hline
\end{tabular}

Yr: years; $n$ : number; IR: Incidence rate; py: person-years. Age data were not available for $n=31$ (0.99\%).

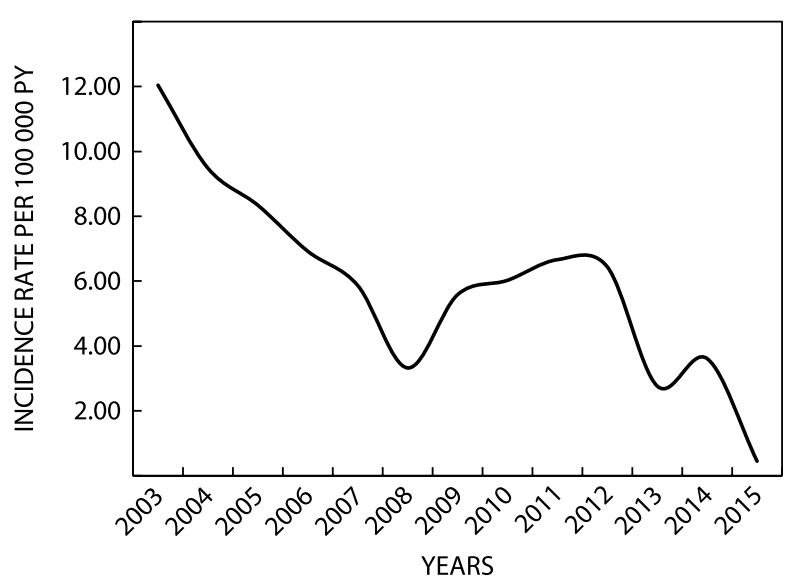

Figure 2: Annual GAS incidence rates among 5256 patients with invasive and non-invasive group A streptococcal infection, Eastern Cape, South Africa, 2003-2015.

Over the study period, there was a decrease in the annual IR (RD: $0.23 / 10^{5}$ py; $95 \% \mathrm{Cl}: 0.02-0.44 / 10^{5}$ py. Breakdown by agespecific categories was $36 \%$ among those $<18$ years of age, $51 \%$ for those aged $18-64$ years, and $13 \%$ for those $\geq 65$ years. The mean age-specific IRs for iGAS were as follows: $<18$ years: $0.38 / 10^{5}$ py; $18-64$ years: $0.52 / 10^{5}$ py; and, $\geq 65$ years: $1.03 / 10^{5}$ py. The IRs remained stable between 2003 and 2012, with slight increases and decreases. The IR for iGAS peaked in 2014, followed by a rapid decline in 2015 .

\section{Non-invasive GAS (Eastern Cape)}

Four thousand eight hundred and twenty-eight (4828) cases making up $92 \%$ of the dataset met with the case definition for non-iGAS. The mean age was 31 years (Range: 2 days - 102 years); GAS isolation for age-specific categories were: $28 \%$ for $<18$ years of age; $58 \%$ for $18-64$ years; and, $14 \%$ for $\geq 65$ years (Table 3 ).

Mean annual incidence rates for non-iGAS were 5.48 cases per $10^{5}$ py. Over the complete study period, there was a decrease in the rate of infections (RD: $11.36 / 10^{5}$ py; $95 \% \mathrm{Cl}: 10.53-12.19 / 10^{5}$ py). The mean age-specific IRs for non-iGAS were as follows (Table 3): $<18$ years: $3.24 / 10^{5}$ py; $18-64$ years: $6.77 / 10^{5}$ py; and, $>65$ years: $13.03 / 10^{5}$ py. A multi-modal shaped curve for incidence was observed; GAS IRs declined steadily from the beginning of the study period to 2008 . This was followed by a slight increase before steadily declining to the end of the study
Table 4: Mann-Kendall trend test and Sen slope estimation for GAS infection in the public sector over a 13-year period (Eastern Cape)

\begin{tabular}{|l|c|c|}
\hline GAS infection & Mann-Kendall & $\begin{array}{c}\text { Sen estimation (median } \\
\text { change }(95 \% \mathrm{CI}))\end{array}$ \\
\hline Invasive GAS & $p=0.2$ & $-0.01(-0.03$ to 0.01$)$ \\
\hline Non-invasive GAS & $p=0.002$ & $-0.65(-0.82$ to -0.50$)$ \\
\hline
\end{tabular}

$\mathrm{Cl}$ : confidence interval.

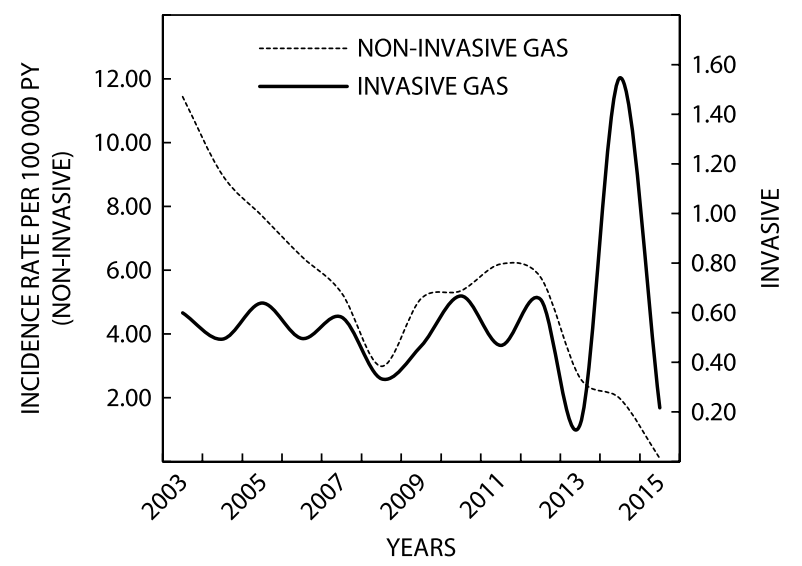

Figure 3: Annual Incidence rates of invasive and non-invasive group A streptococcal (GAS) infection. Eastern Cape, South Africa, 2003-2015.

period. There was a marked decline in the numbers of GAS organisms isolated in 2008 and 2013 (Figure 3). The MannKendall test and the Theil-Sen estimator showed a significant decreasing trend in the incidence of non-iGAS infection over the study period $(p=0.002$ ) (Table 4).

\section{Discussion}

We sought to determine the incidence of GAS infection in all the provinces of South Africa. However, incomplete data were pervasive, with the Eastern Cape province being the only one with information for all the years under review, and accounted for $52 \%$ of the isolates collected in this study. Therefore, the incidence study was confined to the Eastern Cape.

The incidence rates (IR) of laboratory-confirmed $i$ GAS and noniGAS infection reported in the Eastern Cape province of the South African public sector appears to have declined over the last 13 years. Specimens were retrieved from a variety of sources, the bulk of which were pus swabs, blood and urine samples. Of interest, the age-specific IRs increased with increasing age category, with those older than 65 years having the highest IR of 14.06 per $10^{5}$ person-years. Non-iGAS infections showed a statistically significant decrease over the study period.

The laboratory data, retrieved from the NHLS database of South Africa, represent the public health sector serving $84 \%$ of the total South African population. ${ }^{16}$ In the Eastern Cape, the public health sector serves a slightly higher proportion (89\%) of the population in the province than other provinces. ${ }^{17}$ To our knowledge, only two studies have reported incidence data on iGAS in Africa. ${ }^{18,19}$ In a study conducted among children in rural Kenya, the IRs per $10^{5}$ py for all (definite and probable) iGAS were as follows: children <5 years: 35 (95\% Cl: 30 - 40); and, children <1 year: 101 $(95 \% \mathrm{Cl} 83-121) .{ }^{18}$ The second study reported $i$ GAS and noniGAS IRs per $10^{5}$ py: children $<5$ years: 29 ; and, children $<1$ year: 96 
(Cls not stated). ${ }^{19}$ These figures are among the highest reported compared with studies done in other resource limited settings and contrasts noticeably with our low mean IR of 3.62/10 amongst children $<18$ years of age. The high IR reported in Kenya was thought to be due to the presence of a number of risk factors including severe acute malnutrition, malaria and HIV infection which confirm reports from an earlier study conducted in Kenya, where iGAS was associated with malnutrition and HIV infection. ${ }^{19}$ Unfortunately, in our study, data regarding these putative risk factors were unavailable. Elsewhere, in Fiji, high IRs per $10^{5}$ py were also reported: IRs were $26(95 \% \mathrm{Cl} 19-34)$ among those $<5$ years and 85 (95\% Cl 61-116) among those $>65$ years of age. ${ }^{20,21}$ Furthermore, in New Caledonia, the iGAS IR for all ages (definite and probable) was $43 / 10^{5}$ py $(95 \% \mathrm{Cl} 35-52){ }^{22}$ It is possible that the low incidence of GAS infection in our study is due to infrequent submission of specimens for microbiological culture by health practitioners in the Eastern Cape, particularly in more remote areas of the province.

The slight decrease in iGAS incidence in our data is in contrast with those reported elsewhere in the world since the 1980s. ${ }^{23}$ In a surveillance study in Utah, USA, the overall incidence of invasive GAS disease increased significantly from 2002 to $2010 .^{6}$ Ireland also experienced an increase in IRs with the highest annual IR observed in 2013 since iGAS became notifiable in 2004. ${ }^{24}$ Similar increases have been observed elsewhere in Europe. ${ }^{25,26}$ Slightly more males were affected compared with females; and, the increase in incidence of $i$ GAS and non-iGAS disease with age is in keeping with trends seen in studies published in New Caledonia and Fiji. ${ }^{21,22}$

The fluctuations in the IRs, observed over the study period for both iGAS and non-iGAS infection, followed a marked decrease observed in 2008 and 2013 in both iGAS and non-iGAS infection and are not clearly understood. We acknowledge that the variations in the incidence rates may be due to one of two, or both, of the following reasons: (1) it may represent a reflection of the true variance in incidence; or, (2) it may be a reflection in the number of samples submitted for processing by NHLSlaboratories, which may be attributed to the rate of the reporting of cases at any given point in time and/or laboratory budget restrictions. The implications for these would thus be, in the case of the former, an indication that non-iGAS-related diseases are indeed on the decline in the South African setting. However, should the latter be the case with lower numbers of sample submitted to the laboratory, these data would nevertheless, represent the minimum incidence rates for GAS-related diseases. Also, the higher number of GAS cases reported from the Eastern Cape province may be due to close proximity of laboratories to academic hospitals and specimen-taking practices which may vary between the different institutions of the country, rather than a higher prevalence of GAS-related diseases in these provinces. It should also be noted that specimen collection practices may differ among provinces. Also, provinces such as KwaZulu-Natal only joined the digital system in the latter years. Thus, these findings emphasise the need for further research to allow for a more accurate estimation of the extent of GAS-related conditions.

The collection of epidemiological data in many developing countries is poor. A 2005 World Health Organisation report on $i$ GAS disease showed that GAS is an important cause of mortality and morbidity on the global scale and in Africa. ${ }^{3}$ Thus, valid estimates of GAS infection in South Africa, and indeed Africa, are needed to inform the revision of current prevention and control strategies and the development of new primary prevention strategies, including the development of a universal GAS vaccine to reduce the burden of $i$ GAS disease and their consequences. ${ }^{27}$

The main strength of this study is the large sample size of GAS isolates $(>10000)$ that were collected from the NHLS database over a 13-year period from all provinces of South Africa and representing $84 \%$ of the population. It is to be noted that IRs were calculated based on data from the Eastern Cape province only ( $>5000$ ), where NHLS serves $89 \%$ of the population. This study, however, has several limitations including the retrospective design and source of data, and the lack of detailed clinical information in the NHLS database. Moreover, this study may underestimate the incidence of non-iGAS infection, specifically for pharyngitis, given that microbiological throat swabs are not done routinely as standard clinical practice in South Africa. Similarly, for iGAS, our data are likely to underestimate the true incidence given widespread empiric use of antibiotics without culture in clinical practice.

Public health surveillance systems provide valid and reliable scientific information essential to inform appropriate decisionmaking for the best possible action and intervention. ${ }^{8}$ In response to the need for an enhanced surveillance system - to collect high quality data - the AFROStrep registry has been established to collect prospective and comprehensive information on iGAS and non-iGAS infection in Africa as an initial attempt to address the dearth of information on iGAS disease on the continent. ${ }^{10}$

In conclusion, the decrease in IRs among non-iGAS infection and persistent IRs for iGAS infection in the Eastern Cape, emphasises the need to remain vigilant in diagnosing iGAS correctly and for systematically collecting data so as to ensure an efficient and functioning disease-control programme. ${ }^{8}$ Moreover, our data are almost certainly an under-estimate of the true burden of GAS disease which needs to be documented appropriately in a prospective surveillance study proposed by the AFROStrep registry.

Acknowledgements - We appreciate the assistance of the staff at the National Health Laboratory Service Corporate Data Warehouse for carrying out the data extraction.

Declaration - Any opinion, finding, conclusion or recommendation expressed in this material is that of the author(s) and the NRF does not accept any liability in this regard. DDB is supported by a PhD grant from the National Research Foundation of South Africa.

Disclosure statement - None of the authors have a commercial or other association that might have posed a conflict of interest concerning the research presented.

Funding - This work is supported by funding from National Research Foundation of South Africa Thuthuka Programme (TTK14052367799 "The AFROStrep Study"). BMM is funded by the Lily and Ernst Hausmann Research Trust.

\section{ORCID}

Dylan Barth (D) http://orcid.org/0000-0003-1632-5902 Mark E Engel (iD http://orcid.org/0000-0002-1334-8829 Motasim Badri (D) http://orcid.org/0000-0002-5268-6492 Andrew Whitelaw (iD http://orcid.org/0000-0001-9070-5247 Mark E Engel (D) http://orcid.org/0000-0002-1334-8829 


\section{References}

1. Bailie RS, Stevens MR, McDonald E, et al. Skin infection, housing and social circumstances in children living in remote Indigenous communities: testing conceptual and methodological approaches. BMC Public Health. 2005;5:494. doi:10.1186/1471-2458-5-128.

2. Steer AC, Danchin MH, Carapetis JR. Group A streptococcal infections in children. J Paediatr Child Health. 2007;43:203-13. doi:10.1111/ j.1440-1754.2007.01051.x.

3. Carapetis JR, Steer AC, Mulholland EK, et al. The global burden of group A streptococcal diseases. Lancet 2005;5:685-94. https://doi.org/10.1016/S1473-3099(05)70267-X

4. Lamagni T, Efstratiou A, Dennis J, et al. Increase in invasive group A streptococcal infections in England, Wales and Northern Ireland, 2008-2009. Eurosurveillance. 2009;14:1-2.https://www.cabdirect. org/abstracts/20093279880.html (accessed 12 May2014).

5. O'Loughlin RE, Roberson A, Cieslak PR, et al. The epidemiology of invasive group A streptococcal infection and potential vaccine implications: United States, 2000-2004. Clin Infect Dis 2007;45: 85362. doi:10.1086/521264.

6. Stockmann $\mathrm{C}$, Ampofo $\mathrm{K}$, Hersh AL, et al. Evolving epidemiologic characteristics of invasive group A streptococcal disease in Utah, 2002-2010. Clin Infect Dis 2012;55: 479-87. doi:10.1093/cid/cis422.

7. Carapetis JR, Mcdonald M, Wilson NJ. Acute rheumatic fever. Seminar. 2005;366:155-68.

8. Nsubuga $P$, White $M E$, Thacker SB, et al. Public health surveillance: a tool for targeting and monitoring interventions. Washington, DC: World Bank; 2006

9. Robertson KA, Volmink JA, Mayosi BM. Towards a uniform plan for the control of rheumatic fever and rheumatic heart disease in Africa - the Awareness Surveillance Advocacy Prevention (A.S.A.P.) programme. South African Med J 2006;96:241-5.

10. Barth DD, Engel ME, Whitelaw A, et al. Rationale and design of the African group A streptococcal infection registry: the AFROStrep study. BMJ Open. 2016;6: e010248. doi:10.1136/bmjopen-2015-010248.

11. Sharkawy A, Low DE, Saginur R, et al. Severe group A streptococcal soft-tissue infections in Ontario: 1992-1996. Clin Infect Dis. 2002;34: 454-60. doi:10.1086/338466.

12. Invasive Group A streptococcus Sub-Committee. The management of invasive group A streptococcal infections in Ireland; 2006.

13. Su YF, Wang SM, Lin YL, et al. Changing epidemiology of streptococcus pyogenes emm types and associated invasive and noninvasive infections in Southern Taiwan. J Clin Microbiol 2009;47:2658-61. doi:10.1128/JCM.01078-09.
14. Kendall M. Rank correlation methods. 4th ed. London: Charles Griffin; 1975.

15. Birkes D, Dodge Y. 6.9. Estimating the regression line. Alternative methods of regression. New York, NY: J. Wiley \& Sons; 1993. https://doi.org/10.1002/9781118150238

16. Mayosi BM, Benatar SR. Health and health care in South Africa - 20 years after Mandela. N Engl J Med 2014;371:1344-53. doi:10.1056/ NEJMsr1405012.

17. Eastern Cape Department of Health - Annual report 2014/15. 2015;40:1-499.

18. Seale AC, Davies MR, Anampiu K, et al. Invasive group A streptococcus infection among children. Rural Kenya. Emerg Infect Dis. 2016;22:22432. doi:10.3201/eid2202.151358.

19. Berkley JA, Lowe BS, Mwangi I, et al. Bacteremia among children admitted to a rural hospital in Kenya. N Engl J Med 2005;352:39-47. doi:10.1056/NEJMoa040275

20. Steer AC, Jenney AJW, Oppedisano F, et al. High burden of invasive beta-haemolytic streptococcal infections in Fiji. Epidemiol Infect. 2008;136: 621-7. doi:10.1017/S095026880700917X.

21. Steer AC, Jenney A, Kado J, et al. Prospective surveillance of invasive group A streptococcal disease, Fiji, 2005-2007. Emerg Infect Dis 2009;15: 216-22. doi:10.3201/eid1502.080558.

22. Baroux N, D'Ortenzio $E$, Amédéo $N$, et al. The emm-cluster typing system for group A streptococcus identifies epidemiologic similarities across the Pacific region. Clin Infect Dis. 2014;59:e84-92. doi:10.1093/ cid/ciu490

23. Efstratiou A. Group A streptococci in the 1990s. J Antimicrob Chemother. 2000;45(Suppl):3-12.https://www.ncbi.nlm.nih.gov/ pubmed/10759357\%5Cnhttps://jac.oxfordjournals.org/content/45/ suppl_1/3.full.pdf

24. Meehan M, Murchan S, Bergin $S$, et al. Increased incidence of invasive group A streptococcal disease in Ireland, 2012 to 2013. Eurosurveillance. 2013;18:20556. https://doi.org/10.2807/1560-7917.ES2013.18.33.20556

25. Report HP. Group A streptococcal infections: seventh update on seasonal activity, 2013/14. Public Heal Engl. 2014;8.

26. Jessicadarenbergsmise JD, LeppT, Tegnell A, et al. Increased incidence of invasive group A streptococcal infections in Sweden, January 2012 - February 2013. Euro Surveill. 2013;18:3-7.

27. Dale JB, Fischetti VA, Carapetis JR, et al. Group A streptococcal vaccines: paving a path for accelerated development. Vaccine. 2013;31:216-22. doi:10.1016/j.vaccine.2012.09.045 